\title{
Éditorial: Les femmes, L'État-Providence et l'aide
}

On pense que la prochaine crise de l'État-Providence surviendra après 2010 lorsque les "baby-boomers" prendront leur retraite et commenceront à toucher leur pension. Eh bien, c'est faux. La prochaine crise de l'État-Providence sera celle des prestations de soins et services. D'ailleurs, elle sévit déjà. Cette crise du système de soins résulterait de l'accroissement de la demande de services, les personnes âgées étant plus nombreuses et leur longévité accrue. C'est encore faux. Cette crise provient d'une transformation de l'offre. Comme nous le rappelle Alan Walker dans l'article vedette de ce numéro de La Revue canadienne du vieillissement, ce sont les femmes qui assument le gros de l'entraide, donc de la "reproduction sociale".

Si une partie de ces tâches est assurée par des infirmières, des travailleuses sociales et d'autres intervenants des réseaux professionels, il reste qu'aujourd'hui, comme hier, l'essentiel du travail repose sur des filles, des soeurs, des épouses, des mères et des voisines, toutes considérant que leur intervention auprès des hommes et des femmes fait partie de leurs tâches domestiques bénévoles. Dans ce contexte, la crise de la prestation des soins et services provient d'une diminution radicale du temps de bénévolat dont peuvent aujourd'hui disposer des femmes qui ont toujours accompli ce genre de tâches traditionnelles.

Dans le Canada d'aujourd'hui, les systèmes de distribution des soins et des services reposent sur des principes d'organisation du travail et sur des modèles familiaux qui deviennent de plus en plus rares. Au cours des années d'après-guerre, au moment où avaient été mises en place nos institutions sociales actuelles, le temps de travail était partagé dans la famille entre les tâches rétribuées des hommes et le travail bénévole des femmes (éducation des enfants, services aux personnes âgées, activités communautaires variées, etc...). Naguère courant, un tel modèle familial est aujourd'hui exceptionnel. Ainsi, en 1961, les couples avec un seul salaire constituait 65 pour cent de toutes les familles canadiennes. En 1986, ce pourcentage était tombé à 12 pour cent (Canadian Advisory Council on the Status of Women, 1990: 15).

Dans les temps modernes, peu de statistiques montrent des transformations aussi radicales que le développement de la main d'oeuvre féminine, incluant les mères de jeunes enfants. Entre 1980 et 1990, le pourcentage de femmes, mères d'enfants de moins de trois ans et travaillant à l'extérieur, a grimpé de 36,8 pour cent à 53,3 pour cent (Statistics Canada, 1991:A6). En outre, un phénomène moins spectaculaire mais tout aussi important, apparait dans la transformation de la composition des ménages: davantage de ménages d'une seule personne et de familles monoparentales. On peut alors deviner les conséquences frappantes de cette situation sur la répartition du temps au sein des familles et des ménages. Si la quantité d'heures payées a 
baissé pour les individus, elle a considérablement augmenté pour les familles et pour les ménages. Ainsi, après la Seconde Guerre mondiale, il était courant de voir des familles "vendre" 48 heures de leur temps au marché du travail, ce qui correspondait à une semaine normale pour un travailleur masculin employé à plein temps. Aujourd'hui, la norme hebdomadaire varie entre 60 et 80 heures: ce sont les heures cumulées, dans un couple où les deux conjoints travaillent.

Il apparaît évident que l'économie et la société tirent profit de cet accroissement du temps de travail des membres des familles. La recherche d'une plus grande équité pour les femmes est aussi liée à ce phénomène. Mais comme l'ont montré les recherches depuis plus de dix ans, pour les femmes, le prix à payer est lourd. La double journée de la femme, combinant son travail et ses heures de bénévolat domestique constitue une donnée de la vie moderne que l'on connaît bien. Ni les hommes ni les politiques n'ont évolué de façon à s'adapter à cette nouvelle réalité. En cette fin de XXe siècle, la société se trouve donc confrontée à une crise de prestation des services, conséquence directe de la contraction du temps qui jalonne aujourd'hui l'existence de la femme. L'ère des BCBG va bientôt déboucher sur une contraction du temps disponible, résultant de la combinaison du travail salarié avec l'éducation des enfants et le service des hommes. En outre, les divorces et séparations multiplient le nombre de mères monoparentales et ce, pour de longues périodes. Mais ce n'est pas fini. Lorsque le nid se vide, survient une nouvelle contraction du temps: les parents âgés se portent moins bien et commencent à ressentir certaines atteintès de la vieillesse. Les familles étant plus petites, le fardeau devient alors d'autant plus lourd pour les intervenants.

Ce qu'il y a de plus remarquable dans cette situation, c'est que les femmes continuent à assurer autant de soins et de services, même si le prix à payer est énorme: elles refusent des promotions, elles changent de travail, elles passent au temps partiel pour répondre aux besoins de leurs familles immédiates ou même élargies. Selon une étude d'envergure nationale, que j'ai menée au début des années 80 avec mon collègue Wallace Clement, il apparaît que 57 pour cent des femmes âgées de 30 à 49 ans ont signalé une ou plusieurs interruptions de carrière suite à des obligations familiales. Comme Walker le mentionne, ces femmes assurent ces services, même si elles ne se sentent pas en relation étroite ou même réciproque avec les personnes qu'elles aident.

À ce sujet, il est intéressant de comparer la crise de la prestation des services affectant l'existence de la femme contemporaine, avec la crise d'ordre économique des temps passés. Au début du siècle, Rowntree comparaît le déroulement classique de l'existence de la classe ouvrière à ce qu'il appelait un "cycle de vie de pauvreté". À cette époque, et tout le long de son existence, la famille, dirigée par l'homme, connaissait, économiquement parlant, une série de hauts et de bas, correspondant à des fluctuations économiques mais aussi à divers aléas dans des domaines aussi différents que l'éducation des enfants, le chômage, la maladie et la vieillesse. 
Les systèmes modernes de bien-être social, incluant des politiques de pensions, d'assurance-chômage, d'allocations familiales avaient été spécialement conçus pour faire disparaître les aléas économiques qui affectaient le déroulement de l'existence. ${ }^{1}$ Aujourd'hui, les nouveaux défis des politiques sociales consistent à trouver les moyens d'atténuer les hauts et les bas jalonnant le cours de l'existence de la femme contemporaine dans son rôle d'aide.

Mais une question se pose: comment faire face à ces nouveaux besoins? Au cours des annés 80, comme le fait remarquer Walker, on a de plus en plus questionné l'opportunité de se tourner vers l'État "fouineur" pour régler les problèmes des individus et des familles. En même temps, certaines compagnies chefs de file, confrontées à de très hauts taux d'absentéisme et à des problèmes de toutes sortes, avaient mis sur pied des programmes très innovateurs pour aider leurs employés à faire face à leurs responsabilités de support social (voir Kamerman et Kahn, 1987). Cependant, si l'on peut tirer une leçon du passé, c'est que les solutions émanant du secteur privé auront une portée très limitée et, dans le meilleur des cas, seront incomplètes. Les nouvelles pratiques professionnelles, prévues pour venir en aide aux familles confrontées à des responsabilités d'intervenants tendent à se limiter aux grandes sociétés solidement établies et aux employeurs du secteur public. Les firmes de moindre importance, centrées sur le travail avant tout, (celles où sont engagées le plus grand nombre de femmes), ne peuvent se permettre de telles mesures, pas plus, d'ailleurs, que les actuels systèmes de pensions qui ont été conçus dans la même optique. En effet, au cours des années 20, les programmes de "bien-être corporatif," incluant les pensions, ont connu une grande vogue auprès des employeurs d'avant-garde, tant au Canada qu'aux États-Unis. Mais, comme tous les pays en ont vite fait l'expérience, le seul moyen de garantir une pension, si modeste fut-elle, au plus grand nombre de travailleurs, était d'en répartir les coûts dans l'économie par la mise sur pied d'une forme d'assurance-vieillesse, au plan national.

La crise des prestations de soins et services devra faire appel au même type de solutions collectives innovatrices.

Toutefois, Walker nous prévient - et à mon sens, il a raison - qu'une législation nationale ne règlera nullement à elle seule la question de la prestation des soins et services. Leur conception même devra être reformulée à tous les niveaux de la société, particulièrement en ce qui concerne la répartition des tâches sociales selon le sexe. Walker insiste sur le besoin de revoir l'organisation des soins et services et sur celui de jeter des ponts entre les réseaux formels et informels d'aide sociale. En outre, il fait valoir la nécessité de changements dans les comportements et les valeurs professionnels véhiculés par le secteur officiel des soins et services. J'ajouterais même que des changements m'apparaissent nécessaires dans l'êtablissement de normes professionnelles et de pratiques personnelles susceptibles de régulariser l'existence des intervenants, qu'il s'agisse de femmes ou d'hommes.

Des politiques sociales éclairées et des pratiques professionnelles 
améliorées peuvent sans aucun doute faciliter la tâche des aidants et rendre l'existence des femmes plus agréable. Mais, si l'on se fie aux expériences des autres pays, une sorte de révolution apparaît nécessaire pour régler le problème, tant dans le domaine des relations hommes/femmes que dans la distribution du travail selon le sexe. La Suède accorde ainsi aux aidants naturels des périodes prolongées de temps rétribué et prévoit en outre d'autres formes de congés. Cependant, ce sont presque toujours les femmes qui se prévalent de ces mesures, très rarement les hommes. En dépit de ces formules progressives, les hommes suédois n'en font guère plus que les Canadiens, en terme de participation au travail domestique. Là-bas, comme chez nous, les tâches d'aidants naturels sont des tâches de femmes. ${ }^{2}$

Nous nous dirigeons vers le XXIe siècle et dans ce contexte, il est clair que nos modes de pensées traditionnels concernant les politiques sociales et l'État-Providence sont d'une utilité très relative face aux défis qui nous confrontent. Si l'État-Providence contemporain est le produit d'un autre temps, il a toutefois permis d'améliorer la qualité de vie des travailleurs et également des personnes âgées qui, de loin, ont reçu la plus grande part des retombées sociales du développement de l'État.

Mais aujourd'hui, le nouveau grand défi des politiques sociales consiste à trouver des solutions aussi novatrices, pour résoudre les problèmes engendrés par une des activités humaines les plus fondamentales: s'occuper les uns des autres. Par le passé, les savants, les professionnnels et toutes les personnes actives dans le domaine du vieillissement ont exercé un très grand "leadership" et ont fait preuve de beaucoup d'énergie et d'intelligence dans la recherche de solutions aux problèmes aigus de leur temps. Comme le démontre l'essai d'Alan Walker, la tradition continue.

John Myles, Université de Carleton

\section{Notes}

1 Aujourd'hui, l'embauche des femmes constitue une façon très ambigue de stabiliser à long terme les revenus familiaux. À la suite de la faiblissement récent de l'État-Providence, les familles avec deux salaires sont plus essentielles que jamais pour faire face aux périodes de chômage ou de maladie.

2 Ces conclusions sont fondées sur des enquêtes nationales identiques menés au Canada, aux États-Unis, en Suède, et en Norvège (Clement et Myles, à paraître).

\section{Références}

Canadian Advisory Council on the Status of Women. (1990). Women and Labour Market Poverty. Ottawa.

Clement, Wallace \& Myles, John. (À paraître). Relations of Ruling: Class, Gender and Postindustrialism in Comparative Perspective.

Kamerman, Sheila \& Kahn, Alfred. (1987). The Responsive Workplace: Employers and $a$ Changing Labor Force. New York: Columbia University Press.

Statistics Canada. (1991). Labour Force Annual Averages: 1990. Ottawa: Ministry of Supply and Services. 